\title{
Tipo de emprendimiento y fase de desarrollo como factores clave para el resultado de la actividad emprendedora
}

\author{
Manuel Almodóvar ${ }^{1}$
}

Recibido: 15 de noviembre de 2017 / Aceptado: 22 de mayo de 2018

Resumen. No todas las manifestaciones de actividad emprendedora son necesariamente productivas, en términos de crecimiento económico. Por otro lado, el impacto del emprendimiento parece seguir una pauta diferente en función del estado de desarrollo del país en que se aplique, puesto que su impacto es negativo o nulo en las economías en desarrollo, y positivo en las economías desarrolladas.

Es decir, el resultado del emprendimiento depende del tipo de emprendimiento y de la economía en que se aplique. Este artículo pone en cuestión, a partir de una revisión de la literatura científica, la concepción prevaleciente en las políticas públicas que establece una relación directa entre el emprendimiento y el crecimiento económico y reflexiona acerca de la necesidad de diferenciar entre tipos de emprendimiento y tipos de economía, así como sus vínculos"

Palabras clave: Emprendimiento; crecimiento económico; tipo de emprendimiento; fase del desarrollo.

\section{[en] Type of entrepreneurship and country's stage of development as key factors for entrepreneurial outcome}

\begin{abstract}
Not all kinds of entrepreneurship are necessarily productive in terms of economic growth. On the other hand, entrepreneurship show a different role related to a country's stage of development, because it has negative or null effect on developing countries, and positives one on developed countries. In others words, entrepreneurship outcome depends on, not only the type of entrepreneurial activity, but also on country's type of economy. We also provide guidelines for this two issues: how (types of entrepreneurship) and where (type of economy) entrepreneurship should be useful to achieve economic growth.

Using scientific research, this paper cast doubt on the prevailing opinion among politics that entrepreneurship always fuels economic growth
\end{abstract}

Keywords: Entrepreneurship; economic growth; type of entrepreneurship; stage of development.

Sumario: 1. Introducción. 2. Tipos de emprendimiento y su relación con el crecimiento económico. 2.1 Actividad emprendedora por oportunidad y por necesidad. 2.2 Emprendimiento innovador. 2.3 Clasificación de la actividad emprendedora desde la perspectiva institucionalista. 2.4 Actividad emprendedora local y sistémica. 2.5 Actividad emprendedora formal e informal. 3. Tipos de economía y su relación con el emprendimiento. 4. Conclusiones y discusión. 5. Referencias bibliográficas.

Cómo citar: Almodovar, M. (2018) "Tipo de emprendimiento y fase de desarrollo como factores clave para el resultado de la actividad emprendedora". Cuadernos de Relaciones Laborales, 36(2), 225-244.

Universidad de Extremadura. Departamento de Economía Financiera y Contabilidad.

manuelag@unex.es 


\section{Introducción}

El conocimiento científico ha relacionado el emprendimiento, en un sentido amplio, con grandes beneficios económicos. Asumiendo que los frutos de la actividad emprendedora son necesariamente positivos, parte de los investigadores y de los poderes públicos han relegado a un segundo plano el impacto real del emprendiendo, centrándose principalmente en su promoción (Audrestsch, 2009; Almodóvar, 2016b). El problema que genera esta asociación es pensar que el emprendimiento en un sentido amplio, o emprendimiento genérico ${ }^{2}$, puede aplicarse de forma indiscriminada y que irremediablemente desembocará en resultados positivos.

No obstante, ciertos investigadores nos previenen en este sentido. Entre los primeros se encuentran Fadahunsi y Rosa (2002). Los autores advierten que no valorar el tipo de actividad emprendedora que se aplica puede derivar en consecuencias inciertas.

Vivarelli (2016), que representa un ejemplo más reciente de esta idea, desaconseja basarse en el emprendimiento genérico como el principal impulsor del cambio estructural y tecnológico cuando el objetivo es avanzar del estatus de economía de ingresos medios a altos ingresos. En la misma línea, el trabajo de Liñán y Fernández-Serrano (2014) evidenció que la actividad emprendedora, en un sentido genérico, no estaba relacionada con el crecimiento económico, mientras que otro tipo de emprendimiento (por oportunidad) se vinculaba a mayor crecimiento económico.

En consecuencia, parece lógico que algunos estudios se hayan basado exclusivamente en tipos específicos de emprendimiento para valorar su impacto. Por ejemplo, Aparicio et al. (2016) establecen un impacto positivo del emprendimiento basado en la oportunidad sobre el crecimiento económico. Valliere y Peterson (2009) señalan que sólo un tipo específico de emprendedores generan crecimiento económico, descartando explícitamente a los emprendedores en sentido genérico. También Sautet (2013) reconoce al emprendimiento sistémico como una causa del crecimiento económico frente a otros tipos de actividad emprendedora.

En todas estas concepciones subyace la idea de que "la contribución del emprendimiento al desarrollo macroeconómico es contingente al tipo de emprendimiento" (Van Praag y Van Stel, 2013:352). Este aspecto es especialmente importante si tenemos en cuenta que el emprendimiento puede materializarse en actividades destructivas (Desai et al., 2013). En otras palabras, el tipo de emprendimiento importa, y quizá la clave para el crecimiento económico esté más relacionada con el tipo de actividad que se inicia que con el hecho de que se inicie o no una actividad. No obstante, debe matizarse que existen otros factores claves en la actividad emprendedora, tales como la edad, el sexo, el nivel educativo...pero estos no forman parte del objeto del presente estudio.

En otro sentido, aunque también relacionado con el impacto del emprendimiento, existe un grupo de autores que sostienen que la dinámica de la actividad emprendedora está vinculada a la etapa económica del país donde se aplique dicho emprendimiento (Amorós et al., 2012). A grandes rasgos, las economías más desfavorecidas obtienen poco rendimiento del emprendimiento sobre el crecimiento económico pues su impacto es negativo o nulo, mientras que en las economías avanzadas el

Entendido como cualquier acto de emprendimiento, sin tener en cuenta sus características, por lo que no responde a un tipo específico de actividad emprendedora 
emprendimiento beneficia a la economía (Van Stel et al., 2005; Wennekers et al., 2005; Almodóvar, 2016a). En otras palabras, cuando nos referimos a variables como emprendimiento y desarrollo económico, resulta fundamental distinguir la etapa económica (Vivarelli, 2016).

En definitiva, ante la opción de promover el emprendimiento para conseguir el desempeño económico debemos valorar dos aspectos. En primer lugar, parece necesario distinguir si queremos apoyarnos en el emprendimiento genérico o en tipos específicos de actividad emprendedora y, en segundo lugar, resulta conveniente diferenciar el estadio económico en que se encuentra el país en el que vamos a aplicarlo. Es decir, valorar cómo emprender (tipo de emprendimiento) y dónde emprender (etapa económica).

Precisamente, el objetivo del presente trabajo consiste en ofrecer orientación en estas dos cuestiones. Lógicamente, para valorar cómo emprender, se señalarán tipos de actividad emprendedora y su subsiguiente impacto en la economía. Del mismo modo, para arrojar luz sobre la pregunta dónde emprender, se indicarán las distintas clasificaciones de las etapas económicas y su relación con el emprendimiento. En conclusión, se trata de saber qué podemos esperar del emprendimiento en función del relato científico.

Este trabajo se estructura en cuatro partes: la primera es la presente introducción. En la segunda sección se plantean los diferentes tipos de actividad emprendedora y el impacto económico de cada tipología. En la tercera se señalan las etapas económicas y su relación con la actividad emprendedora, y en la cuarta y última parte, se expone la discusión y conclusiones.

\section{Tipos de emprendimiento y su relación con el crecimiento económico}

Tal como expresamos en la introducción, algunos trabajos subrayan la importancia de diferenciar el tipo específico de emprendimiento con el objeto de valorar su impacto. Por este motivo, en la presente sección trataremos de ofrecer una panorámica general sobre ciertas tipologías de actividad emprendedora, así como su repercusión económica.

\subsection{Actividad emprendedora por oportunidad y por necesidad}

El origen de la diferenciación entre actividad emprendedora por oportunidad y por necesidad se encuentra en los informes del Global Entrepreneurship Monitor (Reynolds et al., 2005), y se realiza en función de la motivación del individuo para emprender. Como resultado, se obtienen dos categorías: el emprendimiento por oportunidad y el emprendimiento por necesidad.

La actividad emprendedora por oportunidad ${ }^{3}$ es aquella que se inicia para explotar alguna oportunidad de negocio. En cambio, el espíritu emprendedor por necesi$\mathrm{dad}^{4}$ aparece cuando no existen alternativas de empleo o estas son insatisfactorias (Reynolds et al., 2002; Benz, 2009; Devins, 2009). 
Según nos sugieren las investigaciones, las nuevas actividades por necesidad se emplazan con mayor intensidad en las economías más débiles, mientras que en las economías desarrolladas encontramos más iniciativas por oportunidad. Así, y apoyándose en la motivación, la literatura señala una relación entre el tipo de economía y el tipo de negocio que se crea: los emprendedores motivados por la oportunidad prevalecen en los países de altos ingresos, y los emprendedores motivados por necesidad dominan en los países de bajos ingresos (Amorós et al., 2012).

Si bien es cierto que las economías avanzadas ofrecen niveles bajos de emprendimiento total frente a las economías en desarrollo, también debe señalarse que, en estas economías desarrolladas, las proporciones de iniciativas motivadas por oportunidad son mayores respecto a las motivadas por necesidad (Larroulet y Couyoumdjian, 2009). Por el contrario, los países menos avanzados tienen mayores tasas de actividad emprendedora total, aunque la presencia relativa de iniciativas por oportunidad es menor.

Es importante estudiar el motivo por el que se comienza un negocio, puesto que los resultados (económicos) de la iniciativa empresarial varían en función de la motivación del emprendedor (Hessels et al., 2008; Liñán et al., 2013). Sirva como ejemplo Urbano y Aparicio (2016) y Aparicio et al. (2016). Ambas investigaciones describen una relación positiva entre los nuevos negocios por oportunidad y el crecimiento económico, por lo que califican a este tipo de actividad emprendedora como una posible clave para el crecimiento económico. Además, indican que las actividades por necesidad no tienen efecto a largo plazo en el crecimiento económico a pesar de que pueden tener efectos a corto plazo. Reforzando estos argumentos, Acs y Varga (2005), en su trabajo para 11 países, encuentran que las iniciativas por oportunidad manifiestan un efecto positivo significativo (medido por la difusión de la tecnología) sobre el desarrollo económico, y que el emprendimiento por necesidad no muestra ningún efecto significativo. También Gries y Naudé (2010) proponen que la creación de empresas por oportunidad puede impulsar la transformación estructural mediante la innovación, el suministro de insumos, el aumento del empleo y la productividad.

En función de estos razonamientos podemos apreciar una relación entre el emprendimiento y el crecimiento económico. Por tanto, el siguiente paso consiste en buscar una respuesta que aclare el motivo de esta relación.

Las investigaciones plantean que la respuesta está en la calidad del emprendimiento. En primer lugar, el contexto de los países menos desarrollados impulsa las actividades por necesidad al no existir otras alternativas de subsistencia (Díaz et al., 2013). Por otro, Anokhin y Wincent (2012) señalan que las oportunidades difieren entre los países desarrollados y en desarrollo, y en estos últimos las iniciativas son de mayor calidad (Shane, 2009).

El mayor consenso entre los académicos consiste en destacar el escaso impacto del espíritu emprendedor por necesidad. Éste aparece principalmente como negocios básicos de subsistencia (Valliere y Peterson, 2009), y no genera el conocimiento suficiente para crear crecimiento económico (Sautet, 2013), es decir, el avance hacia el desarrollo representa un alejamiento del espíritu emprendedor por necesidad (Sautet, 2013).

Sin embargo, la clasificación del emprendimiento entre actividades de oportunidad y necesidad ha recibido ciertas críticas. Por ejemplo, Minniti et al. (2006) des- 
tacan que esta tipología tiene un carácter excluyente ${ }^{5}$, pero en la práctica casi todos los emprendedores pueden ser clasificados en ambas categorías simultáneamente. En la misma dirección, Williams y Williams (2014) opinan que ambas motivaciones pueden coexistir en un mismo individuo, por lo que esta categorización resulta simplista y no ofrece un enfoque válido para definir las motivaciones del emprendedor.

Valliere y Peterson (2009) centran su crítica en el emprendimiento por oportunidad. Según estos autores, la creación de empresas por oportunidad no tiene relevancia en el crecimiento económico. Ello se debe a que este tipo de emprendedores no tienen altas expectativas para el futuro de sus empresas porque sus objetivos de crecimiento son más modestos, o porque sus entornos o mercados son más limitados. Como resultado, las iniciativas impulsadas por la oportunidad no son un predictor positivo del crecimiento económico. Aunque esta crítica ataca al papel de la oportunidad en el desempeño económico, se reconoce un rol al emprendimiento por necesidad, en el sentido de que estas iniciativas son las responsables del crecimiento económico escaso.

Ante las críticas expresadas en líneas anteriores, se presenta una posible solución para poder aplicar esta clasificación. Consistiría en utilizar el ratio oportunidad/emprendimiento total, tal como proponen Larroulet y Couyoumdjian (2009). Así, para analizar el impacto de la actividad emprendedora, la variable clave es la proporción de la iniciativa empresarial motivada por la oportunidad al compararla con la totalidad de actividades emprendedoras.

Otra solución propuesta en forma de ratio, quizá más extendida, consistiría en calcular el porcentaje de oportunidad sobre la necesidad ${ }^{6}$. Esta medida indica la proporción de oportunidad respecto a la necesidad que motivan las iniciativas de negocios. Dichas proporciones son consustanciales a cada nivel económico (Acs et al., 2008), por lo que el ratio es menor en las economías menos favorecidas, y mayor en las más desarrolladas. Adicionalmente, este indicador ofrece información sobre la fortaleza de las empresas, pues los países con un bajo ratio de oportunidad/necesidad tienden a tener empresas más débiles (Wennekers et al., 2005).

\subsection{Emprendimiento innovador}

A nivel macroeconómico, el emprendimiento basado en la innovación se considera un factor que impulsa el desarrollo económico (Aparicio et al., 2016; Urbano et al., 2016). A nivel micro, si la motivación subyacente al emprendimiento es la innovación se esperará un mejor desempeño del negocio (Vivarelli, 2016). En la misma línea, al comparar el emprendimiento basado en la necesidad con el basado en la innovación, Urbano y Aparicio (2016) señalaron que es el segundo el que tiene impacto en términos de crecimiento económico.

También Vivarelli (2016) atribuye a los emprendedores basados en la innovación un papel para que los países de ingresos medios avancen hacia el siguiente estadio del desarrollo. Según su trabajo, el emprendimiento innovador puede utilizarse como "un vehículo para fomentar y acelerar un cambio estructural en favor de esos regímenes que pueden considerarse motores para la innovación, la creación de capaci-

Cada emprendedor únicamente puede encuadrarse en una de las dos clasificaciones.

6 Resultado de dividir la tasa de oportunidad entre la tasa de necesidad. Por ejemplo, Liñán et al. (2013) utilizan el ratio oportunidad/necesidad para estudiar el espíritu emprendedor. 
dades y, finalmente, el crecimiento de la productividad y la convergencia" (Vivareli, 2016:178).

Cuando hablamos de la relación entre emprendimiento, innovación y desarrollo económico, "la distinción entre países avanzados y en desarrollo es especialmente importante" (Vivarelli 2016:17), porque en todas las economías existen emprendedores innovadores (Koellinger, 2008), pero la actividad emprendedora innovadora se concentra principalmente en países altamente desarrollados (Urbano et al., 2016), y se encuentra con menos frecuencia en los países menos avanzados Naudé (2011).

En los países desarrollados existe una relación positiva entre los nuevos negocios y la innovación (Aghion et al., 2009), hasta el punto que la innovación es el "principal motor del crecimiento en los países desarrollados" (Aghion, 2017:17), pero en los países en desarrollo esta relación no existe.

Es posible aproximar el concepto de iniciativas innovadoras con iniciativas por oportunidad. Para ello, recientes investigaciones identifican el concepto de emprendimiento innovador a la noción schumpeteriana. En la obra de Schumpeter 1934), donde se expone su Teoría del Desarrollo Económico, el emprendedor genera el proceso de "destrucción creativa", puesto que mediante la introducción de innovaciones (nuevos productos, nuevas formas de gestión, materias primas...) se generan innovaciones con mejores resultados y se destruyen las anteriores producciones (o métodos de producción).

Autores como Vivarelli (2016) o Mthanti y Ojah (2017) argumentan que el emprendedor desempeña el papel de un agente que, mediante la asunción de riesgos, introduce innovaciones en el mercado con el objeto de explotar una oportunidad. Es esta última afirmación la que de algún modo nos permite equiparar el emprendedor por oportunidad al emprendedor innovador, y se ve reforzada por el trabajo de Aparicio et al. (2016), donde se señala que los emprendedores motivados por la oportunidad tienden a basarse más en la innovación.

Dicha concepción genera una polaridad en el emprendimiento debido a que el emprendedor innovador queda claramente diferenciado del emprendedor por necesidad (o defensivo), cuyo estímulo para iniciar un negocio se circunscribe exclusivamente a la supervivencia (Vivareli, 2016).

El emprendimiento innovador, al igual que el imitador, es un fenómeno que se encuentra en todas las economías independientemente de su grado de desarrollo. No obstante, en los países cuyas economías están muy alejadas de la frontera de producción, tales como los países en desarrollo, predomina una mayor proporción de iniciativa imitativa, al contrario que las economías cercanas a su frontera de producción (países avanzados), donde las nuevas actividades tienen más componente innovador.

Los investigadores se han interesado por el papel de los emprendedores imitativos en las economías no desarrolladas. Naudé (2011) expresa que las grandes innovaciones no son necesarias para las economías pobres (con el objetivo de desplazar la frontera de producción), por lo que la ausencia de un espíritu emprendedor innovador no implica una restricción al desarrollo. Esto se debe a que las innovaciones radicales no son esenciales en las economías pobres para impulsar la frontera de producción y la tecnología (a diferencia de las economías desarrolladas). Minniti y Levesque (2010) proponen un modelo de desarrollo económico en el que no es necesario el gasto en investigación y desarrollo. Dicho modelo se apoya en la observación de países emergentes, donde en algunas ocasiones se ha conseguido el 
desarrollo basándose exclusivamente en la imitación con escasas o nulas inversiones en I+D. Así, los emprendedores tienen un papel muy importante en los contextos de desarrollo donde se persiguen las oportunidades imitativas y claramente contribuyen al progreso económico. En este sentido, también Sauet (2013) reconoce la capacidad de la imitación, en concreto a partir de encomendarse a terceros países, para las economías inmersas en la fase impulsada por la eficiencia ${ }^{7}$.

Recapitulando, encontramos un grupo de autores que parecen manifestar un doble consenso respecto a las economías no avanzadas: por un lado, carecen de innovación emprendedora, pero por otro, la innovación no es estrictamente necesaria para el crecimiento económico, ya que se puede conseguir con la imitación o por la implantación de terceros países.

A pesar de lo expuesto en párrafos anteriores, algunas investigaciones plantean un límite al alcance del crecimiento basado en la imitación. Por ejemplo, Anokhin y Wincent (2012) exponen que la creación de empresas es un instrumento ineficaz para conseguir el desarrollo tecnológico en los países menos avanzados. Su argumentación parte de que las iniciativas en estas economías son de baja calidad, y están motivadas por la necesidad (como única alternativa al desempleo). Consecuente, la relación entre los nuevos negocios y la innovación es negativa. En conclusión, fomentar el espíritu empresarial con la esperanza de estimular la innovación es una política pública inadecuada para los países en las primeras etapas de desarrollo (no así para las etapas posteriores). De este modo, Anokhin y Wincent (2012) señalan las ventajas de las grandes empresas frente a las nuevas y pequeñas (tanto para las economías en desarrollo como para las recientemente industrializadas). No obstante, los autores matizan que sus reflexiones se refieren exclusivamente a la consecución del progreso tecnológico, por lo que no debe desalentarse la promoción del emprendimiento cuando los objetivos son otros.

En su investigación sobre países emergentes, Vivarelli (2016) señala que los rendimientos procedentes de la imitación y de la importación de tecnologías extranjeras tienen una naturaleza decreciente que conlleva pérdida de productividad, es decir, supone una limitación para avanzar hacia la siguiente fase del desarrollo económico. Este argumento puede complementarse el trabajo de Aghion (2017), donde se expresa que cuanto más cerca está una economía de la frontera tecnológica, el crecimiento económico depende más de la investigación que de la imitación.

\subsection{Clasificación de la actividad emprendedora desde la perspectiva institucionalista}

Con la obra seminal de Baumol (1990), en la que se describían los tipos de emprendimiento en función del entorno institucional, se han generado diferentes perspectivas de las posibles tipologías de emprendedores enmarcadas bajo el enfoque institucional. En las siguientes líneas describimos algunas de estas clasificaciones y los conceptos que surgen de éstas.

Una de las clasificaciones con mayor repercusión en la literatura científica plantea la división de los emprendedores en tres grupos: emprendedores productivos,

Según Porter et al. (2002), en esta fase la tecnología y el diseño provienen del exterior, y no prevalecen los paradigmas tecnológicos dominantes (Aghion et al., 2009).a 
emprendedores improductivos y emprendedores destructivos. Esta división se apoya en la Teoría Institucional y tiene su máximo referente en la obra de Baumol (1990). Según el autor, cuando un individuo decide embarcarse en una aventura empresarial lo hace buscando su propio beneficio (en un sentido económico, de poder o prestigio). El resultado de esta acción para el conjunto de la economía depende de los incentivos establecidos por las instituciones que prevalecen en la sociedad, y el fruto de esta iniciativa no tiene que ser necesariamente positivo.

Para Baumol (1990), la cantidad de emprendedores es relativamente constante, más la forma en que se distribuyen los emprendedores depende de las instituciones. Así, podrán surgir tres tipos de emprendimiento: el emprendimiento productivo, el emprendimiento improductivo y el emprendimiento destructivo. Las formas productivas de emprendimiento tienen como resultado la creación de riqueza; las improductivas, redistribuyen riqueza pero no la crean ${ }^{8}$; y las destructivas, no sólo no crean riqueza sino que la destruyen ${ }^{9}$. Por su parte, Murphy et al. (1991) distinguen dos categorías: el emprendimiento (agentes innovadores que fomentan el crecimiento) y la búsqueda de rentas (engloba tanto la redistribución de la riqueza como su destrucción).

Una posible crítica a estas perspectivas es que no profundizan en las formas destructivas de la creación de empresas, y dicho aspecto es relevante, pues tal como indican Desai et al. (2013) la literatura ha ignorado el estudio del emprendimiento destructivo a pesar de que en algunos países puede representar la mayor proporción del espíritu emprendedor total, y es la forma más lesiva de emprendimiento (Baumol, 1990).

Douhan y Henrekson (2010) añaden un planteamiento al introducir la idea de "segundas mejores instituciones". De forma intuitiva, podríamos aproximar este concepto a la noción de optar por el mal menor. Consiste en que determinadas circunstancias ciertas opciones improductivas tienen mejores repercusiones que las que en principio deberían ser productivas. Entre otros ejemplos utilizan la corrupción. Ésta puede favorecer al emprendedor y a la economía en general en ciertos casos y ante instituciones débiles.

Reforzando este argumento, la publicación de Estrin y Mickiewicz (2012) concluye que en circunstancias donde que la economía sumergida está generalizada, ésta se institucionaliza y proporciona un orden alternativo al de las instituciones formales, por lo que el efecto sobre la entrada de empresas no es negativo.

Henrekson y Sanandaji (2011) culminan la aplicación de la teoría institucional al emprendimiento. Distinguen entre el resultado de los nuevos negocios (productivos o improductivos) y el comportamiento del emprendedor ante las instituciones (cumplidor, evasor o perturbador). Lo más llamativo de su argumentación es que en función de las instituciones vigentes cualquier tipo de iniciativa puede ser productiva o improductiva. Por ejemplo, una respuesta perturbadora (aquella que impulsa el cambio institucional) puede ser positiva cuando las instituciones son perversas, y negativa sí las instituciones son adecuadas. Evadir las instituciones (que implica reducir su impacto sin modificarlas) en un entorno burocrático asfixiante, quizá sea el único camino para emprender un negocio y que éste sea productivo para la sociedad. Cumplir con las instituciones no necesariamente implicará un impacto productivo,

Típicamente actividades de arbitraje

Por ejemplo, actividades de extorsión. 
puesto que en algunos contextos sociales las instituciones demandan un comportamiento negativo ${ }^{10}$.

Henrekson y Sanandaji (2011) no sólo tienen en cuenta la actividad emprendedora en un sentido empresarial, sino que añaden el espíritu emprendedor político. Bajo su punto de vista, se produce una interacción entre ambas dimensiones del emprendimiento donde las acciones de unos y otros pueden tener una repercusión positiva o negativa. Dicha retroalimentación puede explicar el fenómeno por el que ciertas economías, tradicionalmente estancadas, despegan en un círculo virtuoso de emprendimiento y cambio institucional. Este ciclo (círculo virtuoso) puede surgir de dos formas: a) con creación de reformas políticas productivas que crean oportunidades para los emprendedores productivos, o b) con cambios tecnológicos promovidos por emprendedores económicos que crean oportunidades para los emprendedores políticos productivos. En cualquier caso se crea una secuencia en la que un cambio crea oportunidades para el otro, y la respuesta a este cambio determinará el resultado para la sociedad.

Acemoglu y Robinson (2012) abordan la noción de círculo virtuoso desde la perspectiva de las instituciones inclusivas y extractivas, entendiendo por instituciones inclusivas a aquellas que promueven en el espíritu empresarial, la energía y la creatividad de una sociedad, al contrario que las instituciones extractivas. Estas instituciones inclusivas, más frecuentes en los países ricos, surgen en circunstancias históricas críticas, "cuando una serie de factores debilitan el control de las élites que están el poder" (Acemoglu y Robinson, 2012:389). Una vez instauradas "tienden a crear un círculo virtuoso, un proceso de retroalimentación positivo, lo que aumenta la posibilidad de que estas instituciones persistan e incluso se expandan" (Acemoglu y Robinson, 2012:389).

Pero el resultado de la evolución histórica es circunstancial, por lo que, en vez de surgir instituciones inclusivas, es posible que aparezcan (o se mantengan en el caso de que ya estén instauradas) las instituciones extractivas. Si "el círculo virtuoso crea una tendencia potente para que las instituciones inclusivas persistan, resistan a los retos y se expandan [...] las instituciones extractivas crean fuerzas igualmente fuertes para lograr persistir; es el proceso denominado círculo vicioso" (Acemoglu y Robinson, 2012:391). De este modo "el círculo vicioso se basa en instituciones políticas extractivas y crea instituciones económicas extractivas, lo que a su vez da apoyo a las instituciones políticas extractivas, porque la riqueza económica y el poder compran poder político" (Acemoglu y Robinson, 2012:418).

\subsection{Actividad emprendedora local y sistémica.}

Los conceptos de actividad emprendedora local y sistémica han sido planteados por el trabajo de Sautet (2013), donde se establece que la estructura de recompensas derivada de la teoría institucional no es suficiente para explicar las actividades productivas que aparecen en los países en desarrollo. Apoyándose en la teoría institucional, en la interpretación de señales y cooperación, en la teoría de redes, en las capacidades dinámicas y en la complementariedad de la empresa, este autor desarrolla los conceptos de emprendimiento local y sistémico.

10 Por ejemplo, en las sociedades de los Señores de la Guerra, donde un individuo ostenta el poder en una zona basándose en la fuerza militar y actuando al margen del poder legítimamente establecido. 
A grandes rasgos, el espíritu emprendedor local se caracteriza por la ausencia de economías de escala, división del trabajo, acumulación de capital y grandes transacciones, mientras que la actividad emprendedora sistémica se define como aquella socialmente productiva, que explota las economías de escala, implica la división del trabajo, necesita acumulación de capital y la existencia de grandes transacciones. Al contrario que la iniciativa empresarial local, utiliza grandes redes impersonales.

La esencia diferencial entre el emprendimiento local y el emprendimiento sistémico consiste en las oportunidades disponibles en el mercado que se opera, siendo esta característica más importante que la propia naturaleza del emprendimiento, es decir, no se trata del tamaño o la cantidad de oportunidades que ofrece el mercado, más bien, se refiere al alcance de dichas oportunidades.

El emprendimiento local se encuentra en muchos países de bajos ingresos (en desarrollo). Para esta distribución existen tres motivos. En primer lugar, el emprendimiento local tiene sus raíces en el carácter informal de los mercados, y esto limita el alcance de la iniciativa empresarial proyectándose exclusivamente a nivel local. En segundo lugar, se basa en la explotación de las oportunidades locales, y en tercer lugar, se caracteriza por estructuras orgánicas simples.

Nunca se extiende más allá de pequeños ámbitos geográficos (comunidad o pueblo), pues se apoya en las redes personales (fuertes lazos familiares y de amistad). Esas redes son insuficientes para desarrollar el emprendimiento sistémico, puesto que necesita amplias redes ${ }^{11}$ basadas en vínculos más débiles. Otros motivos para que el emprendimiento local surja en muchos países en desarrollo son la calidad de las oportunidades y la dimensión del mercado. Por un lado, la escasa calidad de las oportunidades que ofrece un contexto de bajo desarrollo limita el recorrido del emprendimiento. Por otro lado, la dimensión del mercado en estas economías supone una imposición del propio ambiente (considerada exógeno por el autor), y crea un obstáculo para el desarrollo.

Todas estas afirmaciones pueden inducir a concluir que el emprendimiento local es exclusivo de los países en desarrollo, o que el sistémico lo es en los países desarrollados, pero la realidad no es taxativa y está sujeta a matizaciones: Sautet (2013) sostiene que en las economías más débiles se pueden experimentar expresiones de emprendimiento sistémico, pero en menor grado que en los países desarrollados, por tanto, el aspecto fundamental es que "en algunos lugares del mundo, la posibilidad de que el emprendimiento local se vuelva sistémico o que se descubran oportunidades sistémicas no existe o es reducida" (Sautet, 2013:394).

"La aparición del desarrollo depende de las condiciones para emprendimiento sistémico" (Sautet 2013:399). Prácticamente con esta cita, queda resumida, desde la visión de Sautet (2013), la importancia del emprendimiento local y sistémico. Tras haber expuesto las características del emprendimiento local, es posible deducir la consecuencia en términos de crecimiento económico: el estancamiento económico o, en el mejor de los casos, el crecimiento lento. Incluso aceptando que el emprendimiento local en algunos casos pueda llegar a ser productivo, su alcance, a lo sumo, será limitado.

Adicionalmente, se podría aventurar que, por la propia dinámica de la economía, las iniciativas locales pudiesen transformarse en sistémicas, dando cierto valor para

Sautet (2013:394) habla de conexiones sistémicas, que permitan "transacciones comerciales entre las personas que no se conocen entre sí, separados sobre un espacio geográfico amplio y en muchas redes de negocios". 
el crecimiento a las primeras, puesto que desembocarían en iniciativas realmente beneficiosas (sistémicas). Pero Sautet (2013) prevé esta circunstancia indicando que no existe un proceso natural de transformación, en el sentido de que el progreso no es un ciclo en el que los negocios locales se transforman en sistémicos, sino que la previsión es que la mayoría de emprendimientos locales no se tornarán sistémicos, en especial en los países en desarrollo.

La argumentación de Sautet (2013) incide fuertemente en las condiciones previas en que se desarrollará el emprendimiento, entendidas como el contexto de una economía. Así, la pobreza y la falta de desarrollo es el resultado de una conjunción de factores que obstaculizan fuertemente el potencial de la economía al dificultar el emprendimiento sistémico. Como ejemplo, puede subrayarse la incapacidad de las economías menos avanzadas para tejer de grandes redes, que dificulta la transferencia de conocimiento el procesamiento de la información.

Por último, esta clasificación podría ofrecer un enfoque que pueda explicar la razón por la que en los países en desarrollo, a pesar de la existencia de iniciativas productivas, no se produce un impacto en términos de crecimiento económico, es decir, el alcance de las oportunidades (o la dimensión del mercado) suponen un obstáculo para el desarrollo, pues su tamaño determina la aparición o no de la actividad emprendedora sistémica.

Debido a las peculiaridades de la tipología local-sistémica, es fácil que estos conceptos se identifiquen con las tipologías anteriormente expuestas. El propio Sautet (2013) se encarga de señalar las diferencias que existen respecto a dichas clasificaciones:

En primer lugar, al comparar la clasificación de Sautet (2013) con el emprendimiento por oportunidad y necesidad, debe indicarse que el emprendimiento local se diferencia del emprendimiento por necesidad en que esta clasificación no responde a la motivación del individuo, sino que depende del alcance de las oportunidades que ofrece el mercado. Por el mismo razonamiento, el emprendimiento sistémico se diferencia de la creación de negocios por oportunidad (alcance de las oportunidades del mercado).

En segundo lugar, también cabe la posibilidad de asimilar el emprendimiento innovador con el emprendimiento sistémico. Si bien ambos comparten el reconocimiento de una oportunidad que explotan comercialmente y que en los dos casos puede convivir algún tipo de innovación, la diferencia fundamental descansa, al igual que en otros casos, en el tipo de oportunidades disponibles del mercado en que se opera. Sirva como ejemplo de esta diferencia la posibilidad de crear un negocio innovador en un contexto con escasas oportunidades, frente a un negocio innovador en un ambiente cuyas oportunidades fuesen de gran alcance. En los dos casos, el emprendimiento sería innovador pero no sistémico.

En tercer lugar, abordaremos las diferencias entre el emprendimiento institucional $^{12}$ y el emprendimiento local-sistémico. En este sentido, Sautet (2013) observa que, en principio, la aparición del emprendimiento local en las economías más desfavorecida se podría explicar en términos de economía institucional (v.g. derechos de propiedad atenuados, regulación gubernamental predatoria e incertidumbre), pero los mecanismos que impiden el emprendimiento sistémico van más allá de las causas institucionales (tales como la cooperación social, la teoría de redes). Además, Sautet

En la versión de Baumol (1990): productivo-improductivo- destructivo. 
(2013) sostiene que la explicación institucional puede haber subestimado la existencia de emprendedores denominados "productivos" en los países poco desarrollados.

\subsection{Actividad emprendedora formal e informal.}

Otra forma de clasificar el emprendimiento es en función del grado de formalidad de las actividades que realiza. Así, es posible definir al emprendedor informal como el "individuo que participa activamente en la gestión de una nueva empresa dedicada a la venta de bienes y servicios legítimos y no se ha registrado ante las autoridades oficiales" (Autio y Fu, 2015:71), mientras que la "iniciativa empresarial formal es la creación de nuevas empresas que están registradas legalmente en un país determinado" (Dau y Cuervo-Cazurra, 2014:670).

La realidad de la investigación científica respecto al estudio del espíritu emprendedor informal está caracterizada por la escasez de publicaciones (Thai y Turkina, 2014). Siguiendo a Autio y Fu (2015), el motivo es que la literatura se ha centrado en el emprendimiento formal debido a que su medición es más sencilla, y es más frecuente en las economías desarrolladas.

El hecho de que se haya estudiado sin la suficiente diligencia no implica que la actividad emprendedora informal sea irrelevante. Si partimos de que la mayoría de pymes en los países en desarrollo se emplazan en el sector informal (Brixiova, 2013), resulta lógico que Autio y Fu (2015) opinen que la comprensión de la iniciativa empresarial informal es básica para el estudio de las economías donde es más frecuente, esto es, en las economías en desarrollo.

También Thai y Turkina (2014) insisten en la envergadura de este tipo de actividad emprendedora, argumentando tres razones: a) es un fenómeno que representa el 30\% de la economía mundial, b) afecta a todos los tipos de economía, y c) es altamente prevalente en ciertos países. Que un negocio sea informal no implica necesariamente que ofrezca bienes y servicios ilegales, es más, pueden comerciar con bienes y servicios lícitos, pero el hecho de no registrarse oficialmente ante el gobierno puede limitar seriamente su crecimiento (Webb et al., 2013).

Mientras que las nuevas actividades económicas formales son típicas de las economías más desarrolladas (Autio y Fu, 2015), el emprendimiento informal se da con mayor frecuencia en las economías con bajos niveles de desarrollo, a pesar de que es un fenómeno que se puede encontrar, en mayor o menor medida, en todas las economías (Thai y Turkina, 2014).

Si bien no es abundante, existe literatura que trata de forma expresa la actividad emprendedora informal. Por ejemplo, el trabajo de Dau y Cuervo-Cazurra (2014) estudia el efecto de las instituciones pro-mercado en el emprendimiento, entendiendo este tipo de instituciones como los marcos regulatorios que permiten que los mercados funcionen adecuadamente, y que se concretan en la liberalización económica y los niveles de gobernanza. Tras diferenciar la parte informal y la formal del espíritu emprendedor, concluyen que la liberación económica influye de forma positiva en ambos tipos de iniciativa empresarial, mientras que la gobernanza tiene un efecto negativo sobre la cantidad de nuevas actividades económicas informales (así como en las totales).

Thai y Turkina (2014), estudian 4 factores y su influencia en la creación de empresas, distinguiendo entre iniciativas formales e informales. Debido a su extensión, 
en la Tabla 1 se resumen las principales conclusiones de su trabajo, indicando cada factor estudiado y su influencia en la creación de empresas.

Tabla 1: Conclusiones de Thai y Turkina (2014)

\begin{tabular}{|c|c|c|}
\hline \multicolumn{2}{|c|}{ Factores } & Influencia en el emprendimiento \\
\hline \multicolumn{2}{|c|}{ Oportunidades económicas } & $\begin{array}{l}\text { Fomenta el espíritu empresarial formal } \\
\text { Desalienta la iniciativa empresarial informal }\end{array}$ \\
\hline \multicolumn{2}{|c|}{ Recursos y capacidades personales } & Desalienta la iniciativa empresarial informal \\
\hline \multicolumn{2}{|c|}{ Calidad de la gobernanza } & $\begin{array}{l}\text { Fomenta el espíritu empresarial formal } \\
\text { Desalienta la iniciativa empresarial informal }\end{array}$ \\
\hline \multirow[t]{2}{*}{ Cultura } & $\begin{array}{l}\text { Basada en el rendimiento } \\
\text { (individualismo y espíritu } \\
\text { competitivo) }\end{array}$ & Fomenta el espíritu empresarial formal \\
\hline & Basada en la solidaridad & Fomenta el emprendimiento informal \\
\hline
\end{tabular}

Fuente: Almodóvar (2016a) a partir de Thai y Turkina (2014).

Thai y Turkina (2014) centran sus conclusiones sobre las economías que no han alcanzado el desarrollo, planteando que en los entornos donde las iniciativas informales representan una proporción elevada de la economía, quizá no sea factible la reducción de dicho tipo de iniciativas, recomendando promover el emprendimiento informal. Por tanto, para estos casos, proponen priorizar las mejoras que impulsen los niveles de gobernanza y aplicar reformas, con el fin de favorecer un tránsito posterior al sector formal.

Para concluir este apartado, señalamos el trabajo de Autio y Fu (2015), en el que se investiga el impacto de las instituciones, tanto políticas como económicas, sobre el espíritu emprendedor formal y sobre el espíritu emprendedor informal. Este trabajo concluye que ambas categorías institucionales, aplicadas de forma conjunta, poseen una gran capacidad para fomentar el emprendimiento formal en detrimento del informal.

\section{Tipos de economía y su relación con el emprendimiento.}

En el apartado anterior de este trabajo hemos abordado la cuestión de cómo emprender a través de las diferentes tipologías de emprendimiento. En el presente apartado nos centramos en la pregunta de dónde emprender, para lo que expondremos los tipos de economías (clasificaciones). La justificación para estudiar emprendimiento en el contexto económico donde se desarrolla, es que el impacto de la actividad emprendedora puede estar condicionado a la etapa económica en la que se encuentre un país. Por tanto, no debe sorprendernos que, a la hora de analizar la actividad emprendedora, muchos investigadores opten por tener en cuenta la etapa económica en la que se encuentra un país. De hecho, cierta parte de la literatura científica señala que la iniciativa emprendedora juega un papel diferente respecto al desempeño económico, dependiendo no sólo de la naturaleza del emprendimiento, sino de la etapa económica donde se desarrolle. Así, en este apartado, trataremos de señalar que re- 
percusiones tiene el emprendimiento según las clasificaciones económicas adoptadas por los investigadores.

Antes de exponer la cuestión anterior, debemos señalar que los criterios utilizados a la hora de clasificar una economía dentro de una etapa o estado de desarrollo son heterogéneos, por lo que coexisten diferentes clasificaciones. No obstante, podemos identificar al menos dos tendenticas: por un lado, una clasificación dualista, y por otro, una triple clasificación.

El primer enfoque (clasificación dualista), consiste en la división en dos etapas, que a grandes rasgos son los países en desarrollo y los países desarrollados ${ }^{13}$. El segundo enfoque (triple división), descansa especialmente ${ }^{14}$ en la clasificación de Porter et al. (2002), donde la etapa económica de un país se define en función de la competitividad, distinguiendo así tres niveles: a) la etapa impulsada por los factores, b) la etapa impulsada por la eficiencia, y c) la etapa impulsada por la innovación.

Tabla 2: Ejemplos de clasificaciones económicas en la literatura científica

\begin{tabular}{|l|l|l|}
\hline Clasificación & \multicolumn{1}{|c|}{ Autores } & \multicolumn{1}{c|}{ Sub-clasificación } \\
\hline \multirow{5}{*}{ Dualista } & Urbano y Aparicio (2016) & No OCDE y OCDE \\
\cline { 2 - 3 } & Almodóvar (2016a) & $\begin{array}{l}\text { Impacto negativo y positivo del } \\
\text { emprendimiento }\end{array}$ \\
\cline { 2 - 3 } & Liñán et al. (2013) & En desarrollo y desarrollados \\
\cline { 2 - 3 } & Pinillos y Reyes (2011) & En desarrollo y desarrollados \\
\cline { 2 - 4 } & Vallerie y Peterson (2009) & Emergentes y desarrollados \\
\hline \multirow{5}{*}{ Triple } & Galvão et al. (2017) & Factor, eficiencia e innovación \\
\cline { 2 - 4 } & Kuckertz et al. (2016) & Factor, eficiencia e innovación \\
\cline { 2 - 3 } & Prieger et al. (2016) & Bajos, medios y altos ingresos \\
\cline { 2 - 3 } & Díaz et al. (2013) & Factor, eficiencia e innovación \\
\cline { 2 - 3 } & Acs y Amorós (2008) & Factor, eficiencia e innovación \\
\hline
\end{tabular}

Fuente: Elaboración propia

La clasificación dualista cobra especial sentido en el campo del emprendimiento a partir de los trabajos empíricos que señalan la existencia de un ajuste "U" directo ${ }^{15}$ entre el emprendimiento y el crecimiento económico. Siguiendo a Almodóvar (2016b), el ajuste representa una función cuadrática cuyo término cuadrático es mayor que cero. Consecuentemente, la función constará de un mínimo global. Los valores previos a este mínimo observarán un comportamiento decreciente, y los valores

13 Según Urbano y Aparicio (2016) esta clasificación también puede manifestarse como países OCDE o no OCDE, o países de altos y bajos ingresos. Almodóvar (2016a) también usa esta clasificación, pero en función del impacto del emprendimiento en el crecimiento económico, dependiendo de si es negativo o positivo.

14 Por ejemplo, Prieger et al. (2016) dividen en tres grupos según sean países de bajos ingresos, de ingresos medios o de altos ingresos.

15 La literatura científica ha identificado otros modelos tales como el Patrón de Onda (Fritsch y Mueller, 2004), el Ajuste L (Carree et al., 2007; Fotopoulos, 2012) o la Curva "U" Inversa (Fritsch y Schroeter, 2011; Van Praag y Van Stel, 2013). Para una descripción más detallada puede consultarse a Almodóvar (2016a:126). 
posteriores, un comportamiento creciente. Así, esta función muestra dos partes diferenciadas: la primera parte indica una relación inversa, y al llegar a un mínimo, la relación cambia a directa. Su aplicación a la relación entre la actividad emprendedora y el crecimiento económico, por tanto, sostendrá que la primera parte de la función indicará una relación inversa entre el emprendimiento el crecimiento económico, $\mathrm{y}$ una segunda parte con relación directa (primera derivada positiva).

Si aplicamos este planteamiento el resultado es una relación negativa en los países de menores ingresos hasta llegar a un punto en el que la relación se torna positiva (coincidiendo con los países de altos ingresos). La hipótesis de ajuste "U" podemos encontrarla desde Wennekers y Thurik (1999), Carree et al. (2002) y Van Stel y Carree (2004), aunque su consolidación, probablemente, se establezca con los trabajos de Wennekers et al. (2005) y Van Stel et al. (2005).

En líneas generales, la consecuencia de esta relación es que el comportamiento de la iniciativa empresarial no sólo varía entre países sino también entre los diferentes estados del desarrollo (Amorós et al., 2012). En este sentido, las economías desarrolladas, en términos de crecimiento económico, reciben un impacto positivo del emprendiendo, frente a las economías en desarrollo, donde el impacto es negativo o nulo (van Stel et al., 2005; Wennekers et al., 2005; Almodóvar, 2016a). También, la forma del ajuste, refleja que la cantidad de emprendedores es mayor en los países menos desarrollados frente a los avanzados (Carree et al., 2002; van Stel et al., 2005; Carree et al., 2007; Acs et al., 2008; Acs y Amorós, 2008). En resumen, la actividad emprendedora será de mayor cantidad y menor calidad en los países en desarrollo, al contrario que en los países desarrollados.

Algunos investigadores utilizan los tipos de actividad emprendedora para explicar el origen de la curva "U”. Así, Liñán et al. (2013) indican que en las economías menos desarrolladas el emprendimiento se encuentra igualmente distribuido entre la iniciativa por necesidad y por oportunidad, pero a medida que las economías crecen, la necesidad cae. Liñán y Fernández-Serrano (2014) explican el comportamiento del ajuste "U" basándose en las oportunidades que ofrece el contexto económico: en los países avanzados existen más oportunidades debido a un sistema económico más complejo, unido a una mayor demanda de productos nuevos y diferenciados. Valliere y Peterson (2009) atribuyen el ajuste a la prevalencia, en las economías en desarro1lo, del emprendimiento motivado por la necesidad. No obstante, para los países desarrollados la mayor proporción de iniciativas por oportunidad no es la explicación, sino el mayor porcentaje de emprendedores de alta expectativa. Para Sautet (2013), tal como se señaló en el apartado 2, las oportunidades del mercado son la causa que explica el comportamiento del ajuste "U", y provocan que el emprendimiento sistémico aparezca a medida que se progresa en la etapa económica, debido a que se mejora el alcance y la calidad de las oportunidades.

Una interpretación adicional, ofrecida por Thai y Turkina (2014), se basa en el carácter formal o informal del emprendimiento. Su argumentación descansa en que las iniciativas informales están generalizadas en las primeras fases económicas, y al avanzar hacia el desarrollo, el emprendimiento formal desplaza al informal porque mejoran los salarios y el nivel de competencia. Cuando se consigue a un nivel de desarrollo económico alto, el emprendimiento en su tipología formal crece, con lo que aumenta también en términos generales.

No obstante, la triple clasificación económica de Porter et al. (2002) ha servido para ofrecer una explicación, quizá más dinámica, para la relación "U” del empren- 
dimiento y el crecimiento económico. En esta línea, Acs et al. (2008) indican que, en la etapa impulsada por los factores, las altas tasas de autoempleo hacen referencia a pequeños negocios, especialmente centrados en los servicios y la manufactura. Al avanzar en la etapa económica (a la fase impulsada por la eficiencia), la cantidad de emprendedores disminuye por el efecto de las inversiones privadas, de la inversión extranjera directa o del gobierno. En este punto, resulta más atractivo trabajar por cuenta ajena que emprender, por esto, se encuentran pocas personas dispuestas a crear empresas. Por último, en la fase impulsada por la innovación se recuperan la iniciativa empresarial por la expansión del sector servicios, por el desarrollo de las comunicaciones y por la elasticidad de sustitución trabajo-capital.

También Díaz et al. (2013), y apoyándose en la triple clasificación, desarrollan una argumentación institucional para aportar una explicación al ajuste "U”. Según los autores, en la etapa basada en los factores la estructura de incentivos impulsa a la población a generar actividades de mera subsistencia porque no existen alternativas de empleo, es decir, el contexto institucional adverso impulsa las iniciativas, pero dichos negocios son de escaso impacto económico. Como consecuencia, la elación entre el emprendimiento y la calidad institucional resulta negativa. Al alcanzar la etapa basada en la eficiencia, se abandonan las iniciativas por necesidad a favor del empleo por cuenta ajena propiciado por la mejora de la calidad institucional, puesto que dicha mejora permite la existencia y el surgimiento de grandes empresas. En la última etapa (basada en la innovación), la actividad emprendedora no sólo crece, sino que su impacto es positivo para el crecimiento. Esto es posible por la existencia de un marco jurídico consolidado, unido a la aparición de nuevas oportunidades.

\section{Conclusiones y discusión.}

En nuestro recorrido por la literatura científica hemos señalado estudios que arrojan dudas sobre el papel emprendimiento genérico en el desempeño económico (Sautet, 2013; Liñán y Fernández-Serrano, 2014; Vivarelli, 2016), a pesar de que existen otras investigaciones que lo reconocen como un medio para conseguir el crecimiento económico (Urbano y Aparicio, 2016; Almodóvar, 2016a).

Dichos planteamientos no son necesariamente antagónicos. Por un lado, existen diversos índices que miden el emprendimiento, y en función del índice que se utilice los resultados de la investigación pueden variar (Stenholm et al., 2013). Por otro lado, es posible que esta disparidad de planteamientos refleje que, bajo ciertas circunstancias, el emprendimiento genérico pueda ser aplicable y que derive en beneficios económicos, a pesar de que, si aplicásemos un emprendimiento específico, el beneficio sería todavía mayor. Esta hipótesis, se basa en la lógica que los emprendimientos específicos denominados por la literatura científica como positivos forman necesariamente parte del emprendimiento genérico (en mayor o menor proporción). En otras palabras, existe la posibilidad de que el emprendimiento genérico sea positivo se debido a la proporción de emprendimiento específico que contiene (Almodóvar, 2016a).

Como ejemplo hipotético, supongamos una economía avanzada, en la que como se ha expuesto en la sección anterior, mantendría una relación positiva entre el emprendimiento genérico y el crecimiento económico. En este caso, podríamos detener 
aquí el análisis y concluir que la actividad emprendedora resultaría positiva para esa economía, incluso en su condición de genérica. Pero podríamos verificar si dichas iniciativas genéricas contienen una proporción de emprendimiento específico lo suficientemente alta, y que este fuese el verdadero responsable de los efectos positivos. De este modo se reconciliarían ambas posturas.

Si esta hipótesis se cumple, la política pública puede elegir si fomentar el emprendimiento genérico de impacto positivo, o el específico de impacto positivo aún mayor. Todo dependerá de la dificultad o el coste de apoyar una u otra dimensión de la de actividad emprendedora, o de los beneficios adicionales más allá de la economía que se generen con una u otra estrategia. Es decir, incluso en el supuesto de que ambas opciones manifiesten un resultado deseado, y presuponiendo que tipo específico alcance aún mejor el resultado, para el poder político puede resultar más viable o interesante la aplicación de medidas que fomenten el emprendimiento "para todos" (genérico) que "para algunos" (específicos).

Otro notable punto de debate es la aplicación del emprendimiento en los países menos favorecidos. Si aceptamos la tesis que sostienen la relación de negativa, nula o de menor impacto, del emprendimiento con el crecimiento económico en dichas economías (Van Stel et al., 2005; Wennekers et al., 2005; Urbano y Aparicio (2016); Almodóvar, 2016a), la consecuencia que se extrae para la política pública del emprendimiento es no utilizar la actividad emprendedora como herramienta de política pública para fomentar la economía en este tipo de países (Fritsch y Mueller, 2004; Wennekers et al., 2005; Valliere y Peterson, 2009; Anokhin y Wincent, 2012), esto es, fomentar el crecimiento económico a través de la creación de empresas puede ser problemático en economías emergentes (Fritsch y Mueller, 2004).

No obstante, y en consonancia con lo expuesto en este trabajo, algunas investigaciones que unen tipos de emprendimiento con tipos de economía señalan que ciertas iniciativas específicas son válidas para mejorar las condiciones económicas en determinadas economías no avanzadas. En esta línea, Lecuna et al. (2017) proponen al emprendimiento por oportunidad como un medio para conseguir el crecimiento económico en las economías en desarrollo. Igualmente, Aparicio et al. (2016) señalan que el emprendimiento por oportunidad en las economías latinoamericanas está vinculado al crecimiento económico. También, Brixiova (2013) sugiere la promoción del emprendimiento productivo en los países en vías de desarrollo.

Como puede observarse, en estos estudios se detalla el binomio expuesto en el presente trabajo: tipo de emprendimiento y tipo de economía. Quizá, esta sea una de las claves en el estudio del emprendimiento: precisar los resultados del emprendimiento en función de las herramientas que vamos a utilizar (tipos de emprendimiento) y dónde vamos a aplicarlas (tipo de economía).

\section{Referencias bibliográficas}

Acemoglu, D. y Robinson, J.A. (2012). "Why Nations Fail”. New York: Crown Publishers. Acs, Z.J. y Amorós, J.E. (2008). "Entrepreneurship and Competitiveness Dynamics in Latin America". Small Business Economics. 31: 305-23.

Acs, Z.J. y Varga, A. (2005). "Entrepreneurship, agglomeration and technological change". Small Business Economics. 24: 323-334. 
Acs, Z.J., Desai, S. y Hessels, J. (2008). "Entrepreneurship, economic development and institutions". Small Business Economics. 31: 219-234.

Aghion, P. (2017). "Entrepreneurship and growth: lessons from an intellectual journey". Small Business Economics. 48: 9-24.

Aghion, P., Blundell, R., Griffth, R., Howitt, P. y Prantl, S. (2009). "The effects of entry on incumbent innovation and productivity". The Review of Economics and Statistics. 91: 20-32.

Almodóvar, M. (2016a). “Actividad emprendedora y crecimiento económico" (tesis doctoral). Universidad de Extremadura. Cáceres, España.

Almodóvar, M. (2016b). “¿Es siempre aconsejable la actividad emprendedora para el crecimiento económico?”. Ekonomiaz. 90: 128-159.

Amorós, J.E, Fernández, C. y Tapia, J. (2012). "Quantifying the relationship between entrepreneurship and competitiveness development stages in Latin America". International Entrepreneurship and Management Journal. 8: 249-270.

Anokhin, S. y Wincent, J. (2012). "Start-up rates and innovation: A cross-country examination". Journal of Business Venturing. 43: 41-60.

Aparicio, S., Urbano, D. y Audretsch, D. (2016). "Institutional factors, opportunity entrepreneurship and economic growth: Panel data evidence". Technological Forecasting \& Social Change. 102: 45-61.

Audretsch, D.B. (2009). "The entrepreneurial society". Journal of Technology Transfer. 34: 245-254.

Autio, E. y Fu, K. (2015). "Economic and political institutions and entry into formal and informal entrepreneurship". Asia Pacific Journal of Management. 32: 67-94.

Baumol, W.J. (1990). "Entrepreneurship: Productive, Unproductive and Destructive”. Journal of Political Economy. 98: 893-921.

Benz, M. (2009). "Entrepreneurship as a non-profit seeking activity". The International Entrepreneurship and Management Journal. 5: 23-44.

Brixiova, Z. (2013). "Modeling productive entrepreneurship in developing countries". Small Business Economics. 41: 183-194.

Carree, M.A.; Van Stel, A.; Thurik, A.R. \& Wennekers, S. (2007). The relationship between economic development and business ownership revisited. Entrepreneurship and Regional Development, 19: 281-291.

Dau, L.A. y Cuervo-Cazurra, A. (2014). “To formalize or not to formalize: Entrepreneurship and pro-market institutions". Journal of Business Venturing. 29: 668-687.

Desai, S., Acs, Z.J. y Weitzel, U. (2013). “A Model of Destructive Entrepreneurship: Insight for Conflict and Postconflict Recovery”. Journal of Conflict Resolution. 57: 20-40.

Devins, D. (2009). "Enterprise in deprived areas: What role for start-ups?" International Journal of Entrepreneurship and Small Business. 8:486-498.

Díaz, J.C., Almodóvar, M., Sánchez, M.C., Coduras, A. y Hernández, R. (2013). “Institutional variables, entrepreneurial activity and economic development". Management Decision. 51: 281-305.

Douhan, R. y Henrekson, M. (2010). "Entrepreneurship and second-best institutions: going beyond Baumol's typology”. Small Business Economics. 20: 629-643.

Estrin, S. y Mickiewicz, T. (2012). "Shadow Economy and Entrepreneurial Entry". Review of Development Economics. 16: 559-578.

Fadahunsi, A. y Rosa, P. (2002). "Entrepreneurship and illegality: insights from the Nigerian cross-border trade". Journal of Business Venturing. 17: 397-429.

Fotopoulos, G. (2012). Nonlinearities in regional economic growth and convergence: the 
role of entrepreneurship in the European Union regions. Annals of Regional Science, 48: 719-741.

Fritsch, M. y Mueller, P. (2004). "The effects of new business formation on regional development over time". Regional Studies. 38: 961-975.

Fritsch, M. y Schroeter, A. (2011). Why does the effect of new business formation differ across regions? Small Business Economics, 36: 383-400.

Galvão, A., Mascarenhas, C., Rodrigues, R.G., Marques, C.S. y Leal, C.T. (2017). “A quadruple helix model of entrepreneurship, innovation and stages of economic development". Review of International Business and Strategy. 27: 261-282.

Gries, T. y Naudé, W. (2010). "Entrepreneurship and structural economic transformation". Small Business Economics. 34: 13-29.

Henrekson, M. y Sanandaji, T. (2011). "The interaction of entrepreneurship and institutions". Journal of Institutional Economics. 7: 47-75.

Hessels, J., Van Gelderen, M. y Thurik, A.R. (2008). "Entrepreneurial Aspirations, Motivations, and Their drivers". Small Business Economics. 31: 323-339.

Koellinger, P. (2008). "Why are some entrepreneurs more innovative than others?" Small Business Economics. 31: 21-37.

Kuckertz, A., Berger, E.S.C. y Mpeqa, A. (2016). "The more the merrier? Economic freedom and entrepreneurial activity". Journal of Business Research. 69: 1288-1293.

Larroulet, C. y Couyoumdjian, J.P. (2009). "Entrepreneurship and Growth A Latin American Paradox?” Independent Review. 14: 81-100.

Lecuna, A., Cohen, B. y Chavez, R. (2017). "Characteristics of high-growth entrepreneurs in Latin America". International Entrepreneurship and Management Journal. 13: 141-159.

Liñán, F. y Fernández-Serrano, J. (2014). "National culture, entrepreneurship and economic development: different patterns across the European Union". Small Business Economics. 42: 685-702.

Liñán, F., Fernández-Serrano, J. y Romero, I. (2013). "Necessity and opportunity entrepreneurship: The mediating effect of culture". Revista de Economía Mundial. 33: 21-47.

Minniti, M. y Levesque, M. (2010). "Entrepreneurial types and economic growth". Journal of Business Venturing. 25: 305-314.

Minniti, M., Bygrave, W. y Autio, E. (2006). "Global entrepreneurship monitor: 2005 Executive report”. London: London Business School.

Mthanti, T. y Ojah, K. (2017). "Entrepreneurial orientation (EO): Measurement and policy implications of entrepreneurship at the macroeconomic level". Research Policy. 46: 724739.

Murphy, K., Shleifer, A. y Vishny, R. (1991). The Allocation of Talent: Implications for Growth". Quarterly Journal of Economics. 106: 503-30.

Naudé, W. (2010). "Entrepreneurship, developing countries, and development economics: new approaches and insights". Small Business Economics. 34: 1-12.

Naudé, W. (2011). "Entrepreneurship is not a binding constraint on growth and development in the poorest countries". World Development. 39: 33-44.

Pinillos, M.J. y Reyes, L. (2011). "Relationship between individualist-collectivist culture and entrepreneurial activity: evidence from Global Entrepreneurship Monitor data". Small Business Economics. 37: 23-37.

Porter, M.E., Sachs, J.D. y McArthur, J.W. (2002). “The Global Competitiveness Report 2001-2002”. New York: Oxford University Press. 16-25.

Prieger, J.E, Bampoky, C., Blanco, L.R. y Liu, A. (2016). "Economic Growth and the Optimal Level of Entrepreneurship". World Development. 82: 95-109. 
Reynolds, P.D., Bygrave, W.D., Autio, E., Cox, L.W. y Hay, M. (2002). “Global entrepreneurship Monitor: 2002 executive report”. Kansas City: Kauffman Center for Entrepreneurial Leadership.

Sautet, F. (2013). "Local and Systemic Entrepreneurship: Solving the Puzzle of Entrepreneurship and Economic Development". Entrepreneurship Theory and Practice. 37: 387-402.

Schumpeter, J. (1934). The theory of economic development: An inquiry into profits, capital, credit, interest, and the business cycle (Vol. 55). Transaction publishers.

Shane, S. (2009). "Why encouraging more people to become entrepreneurs is bad public policy”. Small Business Economics. 33: 141-149.

Stenholm, P., Acs, Z.J. y Wuebker, R. (2013). "Exploring country-level institutional arrangements on the rate and type of entrepreneurial activity. Journal of Business Venturing. 28: 176-193.

Thai, M.T.T. y Turkina, E. (2014). "Macro-level determinants of formal entrepreneurship versus informal entrepreneurship". Journal of Business Venturing. 29: 490-510.

Urbano, D. y Aparicio, S. (2016). "Entrepreneurship capital types and economic growth: International evidence”. Technological Forecasting \& Social Change. 102: 34-44.

Urbano, D., Aparicio, S. y Querol, V. (2016). "Social progress orientation and innovative entrepreneurship: an international analysis". Journal of Evolutionary Economics. 26: 1033-1066.

Valliere, D. y Peterson, R. (2009). "Entrepreneurship and economic growth: Evidence from emerging and developed countries". Entrepreneurship and Regional Development. 21: 459-480.

Van Praag, M. y Van Stel, A. (2013). "The more business owners, the merrier?" The role of tertiary education. Small Business Economics. 41: 335-357.

Van Stel, A. y Carree, M.A. (2004). "Business Ownership and Sectoral Growth. An Empirical Analysis of 21 OECD Countries". International Small Business Journal. 22: 389-419.

Van Stel, A., Carree, M.A. y Thurik, A.R. (2005). "The Effect of Entrepreneurial Activity on National Economic Growth". Small Business Economics. 24: 311-322.

Vivarelli, M. (2016). "The middle income trap: a way out based on technological and structural change". Economic Change and Restructuring. 49: 159-193.

Webb, J.W., Bruton, G.D., Tihanyi, L. y Ireland, R.D. (2013). "Research on entrepreneurship in the informal economy: Framing a research agenda". Journal of Business Venturing. 28: 598-614.

Wennekers, A.R.M. y Thurik, A.R. (1999). "Linking Entrepreneurship and Economic Growth". Small Business Economics. 13: 27-55.

Wennekers, S., Van Stel, A., Thurik, A.R. y Reynolds, P.D. (2005). "Nascent entrepreneurship and the level of economic development". Small Business Economics. 24: 293-309.

Williams, N. y Williams, C.C. (2014). "Beyond necessity versus opportunity entrepreneurship: some lessons from English deprived urban neighbourhoods". International Entrepreneurship and Management Journal. 10:23-40. 